REPLY

\title{
Only the PAM explains the personalized nature of empathy
}

\author{
Stephanie D. Preston and Frans B. M. de Waal
}

Responding to our Review (Mammalian empathy: behavioural manifestations and neural basis. Nat. Rev. Neurosci. 18, 498-509; 2017) ${ }^{1}$, Rizzolatti and Caruana (Some considerations on de Waal and Preston review. http:// dx.doi.org/10.1038/nrn.2017.139(2017)) $)^{2}$ state that their "mirror mechanism" is "not complementary to the PAM, nor a subpart of it". Here, we address their concerns and argue that the perception-action model (PAM) is a broad concept that encompasses the mirror mechanism but also takes into account the deeply personal way that empathy depends on personal experience.

We agree, as explained in our review, that mirror neurons and the frontoparietal mirror system (IFG-rPPC; Inferior frontal gyrusrostral posterior parietal cortex, with interconnections to the insula) are important for more than motor behaviour. Indeed, we cited evidence that mirror neurons may be found throughout the brain, that they represent goals and feed back to produce resonant states and that the mirror system supports the representation and imitation of emotional expressions, emotional empathy and empathic pain. In view of these data, we concluded that both the PAM and mirror theories "presume that motor acts and affective states can transfer from a target to an observer in a bottom-up, goal-relevant manner through shared representations for perception and action". Given this agreement, it is unclear why evidence for shared affective representations in the insula, amygdala and posterior part of the anterior cingulate cortextex (pACC) demonstrates a mirror mechanism that is distinct from the PAM. The PAM has always been based on exactly this sort of evidence ${ }^{3-7}$, and these regions were covered in our review ${ }^{1}$. Thus, the mirror mechanism referred to by Rizzolatti and Caruana in their correspondence appears to be encompassed in the affective PAM we described in our review and in previous published papers.

Moreover, the PAM is significantly broader and provides a deeper explanation of empathy than the mirror mechanism because it is the only model that explains how distributed representations develop through the observer's experience (such as memories and associations for the target, situation and state) to produce the individual variation in empathy observed in real life ${ }^{3-5,7-10}$. If a man cries out in pain, doubling over after a ball hits him below the waist, an observing man will feel a more embodied, specific empathic response than a woman. By contrast, a woman performing a similar gesture due to menstrual cramps would elicit a more empathic response from an observing woman, scaled to the degree that she had suffered similarly. Shared experiences are essential for this dimension of empathy. The PAM encompasses this key aspect of the phenomenon by adding distributed, personspecific, conceptual, associative and affective representations to the overlapping representations of self and other. No other neural model - including the mirror mechanism - explains this core component of empathy.
We are pleased that nearly everyone working in this area agrees that empathy depends on neural overlap in motor and affective representations of self and other, but the PAM is broader because it explains how an observer's unique past experience explains the degree that they empathize with the other. Empathy is a deeply personalized phenomenon, and any model that fails to address this does not capture its essence.

Stephanie D. Preston is at the Department of Psychology, University of Michigan, 530 Church Street, Ann Arbor, Michigan 48109, USA.

Frans B. M. de Waal is at the Psychology Department and Living Links, Yerkes National Primate Research Center, Emory University, 36 Eagle Row, Atlanta, Georgia 30322, USA.

Correspondence to S.D.P. prestos@umich.edu

doi: $10.1038 / \mathrm{nrn} .2017 .140$ Published online 3 Nov 2017

1. de Waal, F. B. \& Preston, S. D. Mammalian empathy: behavioural manifestations and neural basis. Nat. Rev. Neurosci. 18, 498-510 (2017).

2. Rizzolatti, G. \& Caruana, F. Some considerations on de Waal and Preston review. Nat Rev, Neurosci. http:// dx.doi.org/10.1038/nrn.2017.139 (2017).

3. Preston, S. D. \& de Waal, F. B. M. Empathy: its ultimate and proximate bases. Behav. Brain Sci. 25 1-20; discussion 20-71 (2002).

4. Preston, S. D. in Empathy in Mental IIIness (eds Farrow, T. F. D. \& Woodruff, P. W. R.) 428-472 (Cambridge Univ. Press, 2007).

5. Preston, S. D. et al. The neural substrates of cognitive empathy. Soc. Neurosci. 2, 254-275 (2007).

6. Buchanan, T. W. et al. The empathic, physiological resonance of stress. Soc. Neurosci. 7, 191-201 (2012).

7. Preston, S. D. \& Hofelich, A. J. The many faces of empathy: parsing empathic phenomena through a proximate, dynamic-systems view of representing the other in the self. Emotion Rev. 4, 24-33 (2012).

8. Preston, S. D. \& Hofelich, A. J. Understanding empathy by modeling rather than organizing its contents. Emotion Rev. 4, 38-39 (2012).

9. Preston, S. D., Hofelich, A. J. \& Stansfield, R. B. The ethology of empathy: a taxonomy of real-world targets of need and their effect on observers. Front. Hum. Neurosci. 7, 488 (2013).

10. Mohr, A. H., Kross, E. \& Preston, S. D. Devil in the details: effects of depression on the prosocial response depend on timing and similarity. Adapt. Hum. Behav. Physiol. 2, 281-297 (2016).

Competing interests statement

The authors declare no competing interests. 